\title{
LES of Asymmetric Wave-induced Flow
}

\author{
J. B. Chen ${ }^{1}$, J. F. Zhou ${ }^{1, *}$, Q. Zhang ${ }^{2}$ \\ ${ }^{1}$ Key Laboratory of Environmental Mechanics, Institute of Mechanics, Chinese Academy of Sciences, Beijing 100190, \\ China \\ ${ }^{2}$ School of Aerospace Engineering, Beijing Institute of Technology, Beijing 100081, China
}

Email: zhoujf@imech.ac.cn

\begin{abstract}
Asymmetric water wave is a common phenomenon in coastal areas, which has a great influence on sediment transport and coast protection. However, previous studies mainly focused on symmetric wave motion. Therefore, it is of practical importance to manifest flow characteristics in asymmetric wave field. To begin with, we have formulated an LES model, by which we carry out numerical simulation of laminar flow induced by asymmetric waves showing reasonable agreement with analytical solution. Then, we apply the model to the cases of asymmetric waves in turbulent flow region. Numerical results of velocity profile and shear stress are obtained and compared with experimental data. We find that, in the case of asymmetric wave, the effect of Reynolds number and the degree of asymmetry are two crucial factors for governing the flows.
\end{abstract}

Key words: large-eddy simulation, asymmetric wave, the degree of asymmetry, Reynolds number

\section{INTRODUCTION}

Asymmetric water wave motion is a common phenomenon in coastal areas, which plays a significant role in sediment transport and coast protection. Previous studies have mainly focused on symmetric wave motion [1-3]. However, nonlinearity of waves becomes more predominant in shallow area, which results in asymmetric characteristic of waveinduced velocity field. Because of this reason, some experimental and theoretical studies have been carried out on turbulence structure in an asymmetric wave boundary layer [4,5]. At the same time, some numerical models have been built to simulate asymmetric wave motion, such as $k$ - $\varepsilon$ model [6], $\sigma$-coordinate 3D model [7] and BSL $k$ - $\omega$ model. Most of these researches are restricted to low Reynolds number. In the present paper, our purpose is to explore flow characteristics of asymmetric waves at high Reynolds number. The large-eddy simulation approach with Smagorinsky subgrid model is adopted to a number of cases. The effect of Reynolds number and the degree of asymmetry on velocity profile and wall shear stress are discussed.

\section{PHYSICAL MODEL}

For asymmetric waves, we define the degree of asymmetry of the wave as follows

$A s=\frac{U_{\mathrm{c}}}{U_{\mathrm{c}}+U_{\mathrm{t}}}$

where $U_{\mathrm{c}}$ and $U_{\mathrm{t}}$ are the velocity magnitudes at wave crest and trough, respectively. Hence, we have $A s=0.5$ for symmetric waves.

Reynolds number $R_{\delta}$ defined by Tanaka and Sumer et al. [8] is used to discuss the flow regime as given below,

$R_{\delta}=\frac{U_{\mathrm{c}} \delta}{v}$ 
Here $\delta=\sqrt{2 v / \omega}, v$ is the kinematic viscosity of fluid, and $\omega$ is the angular frequency of wave.

The computational model is sketched in Figure 1. The flow is driven by a periodic bottom plate oscillation, which results in a cnoidal variation of the free stream velocity. Table 1 lists the parameters of cnoidal waves used in the current study.

Table 1. Parameters of cnoidal waves.

\begin{tabular}{ccc}
\hline Case & $R e_{\delta}$ & $A s$ \\
\hline CN1 & 392 & 0.69 \\
CN2 & 936 & 0.62 \\
CN3 & 700 & 0.69 \\
CN4 & 1000 & 0.69 \\
CN5 & 1500 & 0.69 \\
CN6 & 2000 & 0.69 \\
CN7 & 2500 & 0.69 \\
CN8 & 3000 & 0.69 \\
\hline
\end{tabular}

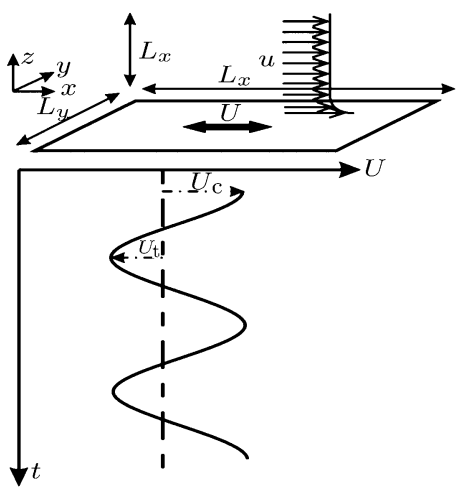

Figure 1: Sketch of the physical model

\section{RESULTS AND DISCUSSION}

1. Laminar flow Tanaka \& Sumer et al. [8] derived the velocity and wall shear stress of laminar boundary layer under cnoidal wave as follows

$$
\begin{aligned}
& u=\frac{U_{\mathrm{c}}}{B_{N}} \sum_{n=1}^{N} a_{n}\left[\cos (n \omega t)-\mathrm{e}^{-\beta_{n} z} \cos \left(n \omega t-\beta_{n} z\right)\right] \\
& \tau=\rho U_{\mathrm{c}} \sqrt{\omega v} \frac{1}{B_{N}} \sum_{n=1}^{N} \sqrt{n} a_{n} \cos \left(n \omega t+\frac{\pi}{4}\right)
\end{aligned}
$$

where $\beta_{n}=\sqrt{n \omega /(2 v)}, B_{N}$ and $a_{n}$ are coefficients that can be obtained from the series expressing cnoidal function.

By introducing dimensionless parameters $u^{*}=U / U_{\mathrm{c}}, t^{*}=\omega t$ and $z^{*}=z / \delta$, the dimensionless velocity and wall shear stress can be obtained. Hereafter $\tau$ is the dimensionless wall shear stress.

Figure 2 shows that the numerical and analytical velocity profile results agree with each other very well. The overshooting velocity is also well predicted. As shown in Figure 3, the comparison between the numerical and analytical wall shear stress shows almost no any error in the whole cycle. From Figures 2 and 3, it may be observed that the velocity profile and the wall shear stress distribution are asymmetric, while those of the symmetric wave are antisymmetric.

2. Turbulent flow Figure 4 shows the phase-variation of velocity profile in intermittent turbulent flow. The numerical results agree with experimental data satisfactorily. Discrepancies exist near the wall, which may be partly owing to the measuring difficulties in the near wall region. Additional case studies demonstrate that the degree of asymmetry has a great impact on velocity profile. The larger the degree of asymmetry is, the more the velocity profile deviates from the symmetric one.

Figure 5 compares time-variation of wall shear stress between numerical and experiment data computed by log-fit method. The present model predicts the overall process reasonably.

The current LES has been applied to higher Reynolds numbers to achieve a fully turbulent boundary layer. Figure 6 shows the wall shear stress for $A s=0.69$ with Reynolds number from 392 to 3000 . It is obvious that the maximum amplitude of wall shear stress increases with Reynolds number. But this dependency seems weak when Reynolds number exceeds 2000 . At $R e_{\delta}=1000$, a sudden decrease in the wall shear stress appears in crest and trough, showing the beginning of transitional behavior of the boundary layer. In addition, the amplitude of wall shear stress of asymmetric wave is larger than that of symmetric wave at the same Reynolds number. 


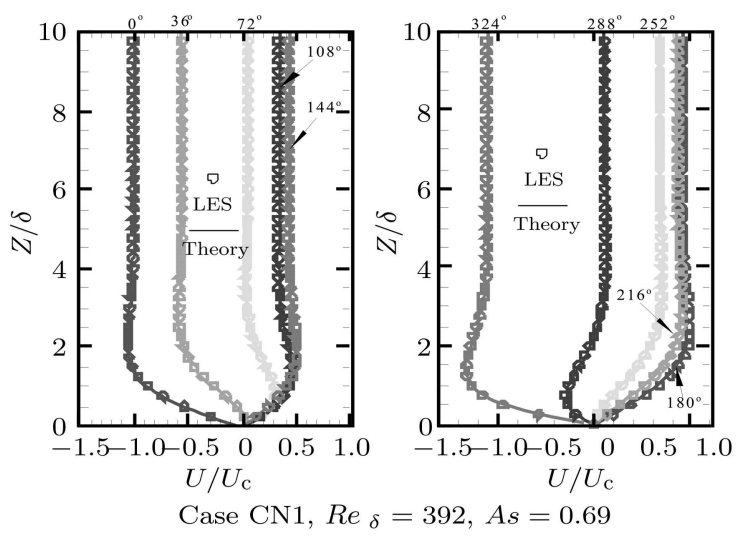

Figure 2: Phase-variation of mean velocity profile

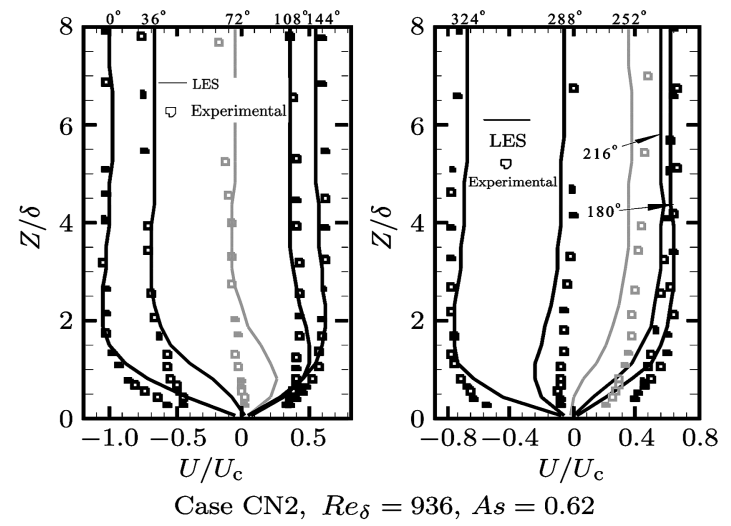

Figure 4: Phase-variation of mean velocity profile

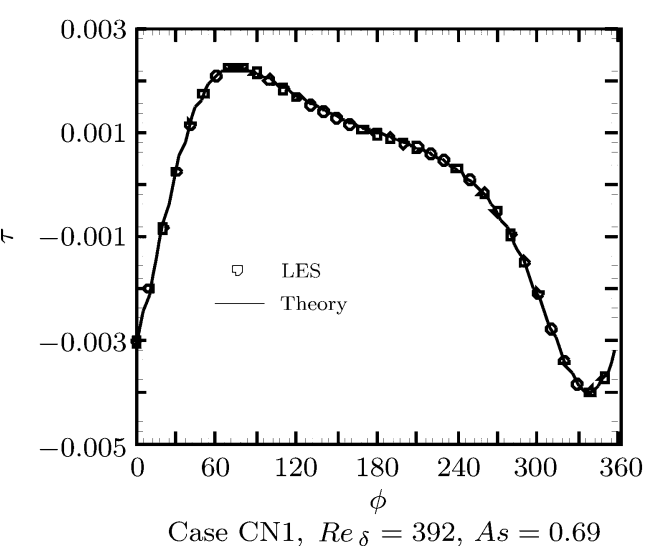

Figure 3: Time-variation of wall shear stress

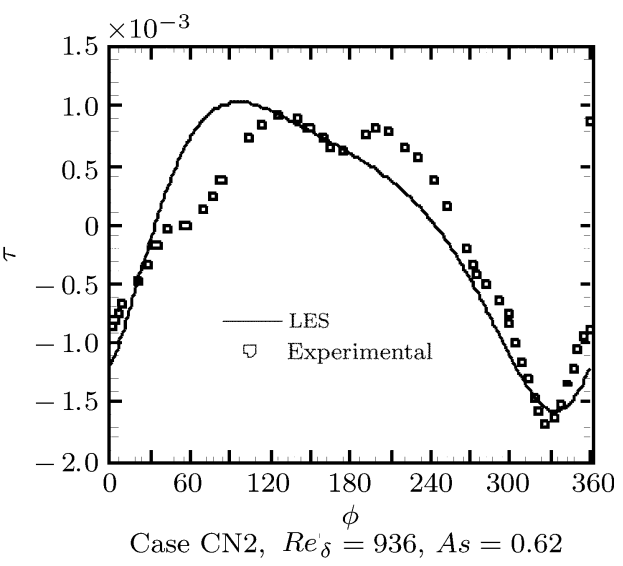

Figure 5: Time-variation of wall shear stress

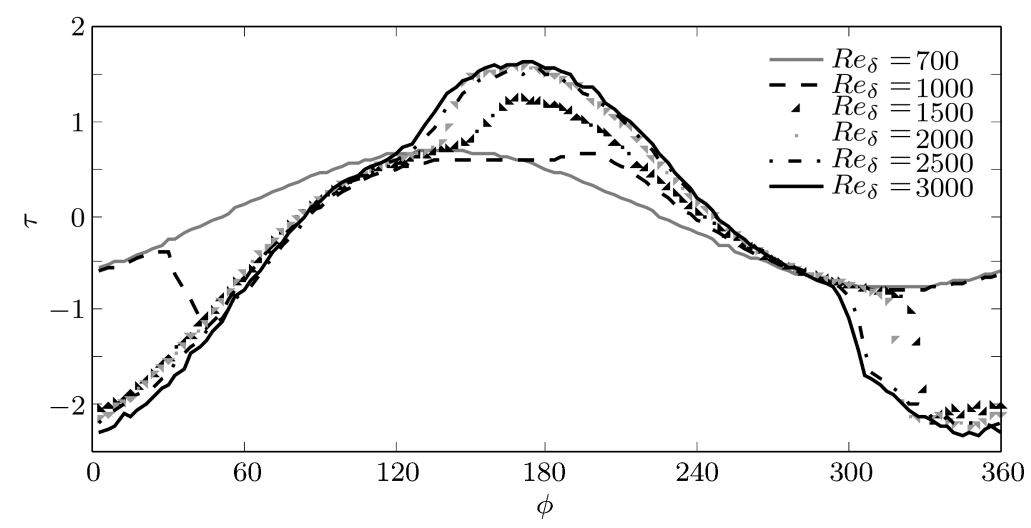

Figure 6: Phase-variation of wall shear stress $(A s=0.69)$

\section{CONCLUSIONS}

In the present study, the large-eddy simulation method has been applied to simulate the laminar and turbulent boundary layer behavior under asymmetric wave. The following conclusions can be drawn: (1) The numerical results of LES approach agree well with the analytical solution for velocity profile and wall shear stress of laminar flow and experimental data of intermittent turbulent flow. In intermittent turbulent flow, there is slight discrepancy between numerical results and experiment data in velocity profile near the wall. This may arise partly from the experiment measure data. (2) The LES model can efficiently predict the mean velocity profile and wall shear stress at higher Reynolds numbers, which can not be achieved by experiment. (3) The effect of Reynolds number and the degree of asymmetry are two crucial factors governing the behavior of asymmetric wave boundary layer. The velocity profile and the wall shear stress distribution of asymmetric wave are asymmetric, while those of the symmetric wave are antisymmetric. The maximum amplitude of wall shear stress increases with Reynolds number. But this dependency seems weak when Reynolds number exceeds 2000. 


\section{Acknowledgements}

We would like to give thanks to the financial support from the National Natural Science Foundation of China (11042003 and 10932012).

\section{REFERENCES}

1. Vittori G, Verzicco R. Direct simulation of transition in an oscillatory boundary layer. J. Fluid Mech., 1998; 371: 207-232

2. Thais L, Chapalain G, Smaoui H. Reynolds number variation in oscillatory boundary layers. Part I. Purely oscillatory motion, Coast. Eng., 1999; 36: 111-146

3. Salon S, Armenio V, Crise A. A numerical investigation of the Stokes boundary layer in the turbulent regime. $J$. Fluid Mech., 2007; 570: 253-296

4. Tanaka H, Sana A, Yamaji H, et al. Experimental and numerical investigation on asymmetric oscillatory boundary layers. J. Hydrosci. Hydr. Eng., 1998; 16(1): 117-126

5. Sana A, Tanaka H, Yamaji H, et al. Hydrodynamic behavior of asymmetric oscillatory boundary layers at low Reynolds numbers. J. Hydraul. Res., 2006; 132: 1086-1096

6. Jones W P, Launder B E. The prediction of laminarization with a two equation model of turbulence. Int. J. Heat Mass Transfer., 1972; 15: 301-314

7. Lin Pengzhi, Li C W. A $\sigma$-coordinate three-dimensional numerical model for surface wave propagation. Int. J. Numer. Meth. Fluids., 2002; 38: 1045-1068

8. Tanaka H, Sumer B M, Lodahl C. Theoretical and experimental investigation on laminar boundary layers under cnoidal wave motion. Coast. Eng. J., 1998; 40(1): 81-98 